\title{
Comparative Study of Demographic Profile, Mortality, Risk Factors, and Bacteriological Profile of Respiratory Isolates from Ventilated Patients: Ventilator-Associated Event versus NonVentilator-Associated Event Cases
}

\author{
Abha Sharma, Madhusmita Das, Bibhabati Mishra, Poonam Sood Loomba \\ Departments of Microbiology, GIPMER, New Delhi, India
}

\section{Abstract}

Introduction: Ventilator-associated pneumonia (VAP) rate has long been considered a quality indicator (QI) in intensive care units (ICUs) for mechanically ventilated patients. However, ever since the Centers for Disease Control and Prevention (CDC) redefined VAP as ventilator-associated events (VAEs), its validity as a tool for use in quality assurance needs to be assessed. Aim: The aim of the study was to identify VAE rate in ICU, compare the demographic and respiratory isolate profile and mortality with non-VAE cases, and identify risk factors for VAE. Materials and Methods: All ICU patients on mechanical ventilation (MV) for $>2$ days were followed prospectively. VAE data were collected using a checklist obtained from the CDC website (the National Healthcare Surveillance Network VAE surveillance tool). The demographic profile, risk factors, and treatment for each patient were recorded in a proforma. VAE rates were estimated per 1000 ventilator-days. Results: The overall VAE rate was 38.1/1000 MV days. Among VAE subtypes, $18.7 \%$ were ventilator-associated condition (VAC), $43.7 \%$ were infection-related VAC (IVAC), and 37.5\% were possible VAP (PVAP). Survival rate was 100\% for patients with VAC alone, whereas only $14.2 \%$ and $16.6 \%$ of patients survived with IVAC and PVAP, respectively. No significant association was found between age, gender, mortality, bacterial isolate, and VAE cases. Depressed level of consciousness, immunosuppression, antibiotic use, and reintubation were significant risk factors associated with VAE cases $(P=0.0001)$. Conclusion: There is no significant difference observed between VAE and non-VAE cases regarding demographic profile, respiratory bacterial profile, and mortality. More research is required to establish the role of VAE as a QI for patient care in ICUs.

Keywords: Intensive care unit, nonventilator-associated events, risk factors, ventilator-associated events

\section{INTRODUCTION}

In 2013, the Centers for Disease Control and Prevention (CDC) redefined ventilator-associated pneumonia (VAP) as ventilator-associated events (VAEs) and as a result the National Healthcare Surveillance Network (NHSN) introduced the new VAE surveillance definition algorithm that recognized a broader range of pulmonary complications, both infectious and noninfectious, that occurred in mechanically ventilated adult patients. ${ }^{[1]}$ The clinical definition of VAP lacks sensitivity, specificity, objectivity and is less accurate. The newer criteria are more specific and objective. ${ }^{[2]}$ Studies done so far have shown that most of the VAEs are due to pneumonia, atelectasis, acute respiratory distress syndrome (ARDS),

\begin{tabular}{|l|l|}
\hline \multicolumn{2}{|c|}{ Access this article online } \\
\hline Quick Response Code: & Website: \\
\hline & www.ijrc.in \\
\cline { 2 - 2 } & \\
\hline
\end{tabular}

and fluid overload. ${ }^{[3]} \mathrm{A}$ VAE is defined as 2 days of stable or decreasing ventilator settings, followed by 2 days of increased ventilator settings (ventilator-associated condition [VAC]). Further subclassification of VAE includes infection-related VAC (IVAC) and possible VAP (PVAP). ${ }^{[1,4]}$ Hence, using

Address for correspondence: Dr. Abha Sharma, Department of Microbiology, GIPMER, New Delhi, India. E-mail: abha_sh79@rediffmail.com

This is an open access journal, and articles are distributed under the terms of the Creative Commons Attribution-NonCommercial-ShareAlike 4.0 License, which allows others to remix, tweak, and build upon the work non-commercially, as long as appropriate credit is given and the new creations are licensed under the identical terms.

For reprints contact:WKHLRPMedknow_reprints@wolterskluwer.com

How to cite this article: Sharma A, Das M, Mishra B, Loomba PS. Comparative study of demographic profile, mortality, risk factors, and bacteriological profile of respiratory isolates from ventilated patients: Ventilator-associated event versus nonventilator-associated event cases. Indian J Respir Care 2021;10:66-9.

Received: 14-03-2020

Revised: $13-07-2020$

Accepted: 06-08-2020 Published: $31-01-2021$ 
the new VAE surveillance criteria, we conducted this study with the aim of identifying VAE rate in our intensive care units (ICUs) and comparing the demographic characteristics and bacteriological profile of isolates obtained from respiratory samples of VAE cases with the non-VAE cases.

\section{Materials and Methods}

This prospective, observational study was conducted with the approval of the ethics committee of the institute after obtaining consent as per institutional policy. All patients who were on mechanical ventilation (MV) for $>2$ days in the ICU were followed up prospectively till they were extubated or expired. The VAE data were collected using a checklist obtained from the CDC website (NHSN VAE Surveillance tool). Demographic profile, risk factors, and treatment for each patient were recorded in a proforma. VAEs were defined according to the new NHSN VAE surveillance algorithm:

- VAC: Increase in the daily minimum positive endexpiratory pressure of at least $3 \mathrm{cmH}_{2} \mathrm{O}$ for at least 2 days or increase in the daily minimum fraction of inspired oxygen of at least 20 points for at least 2 days

- IVAC: Any one of the following four criteria - fever or hypothermia, leukocytosis, or leukopenia, and new antimicrobial agent started and continued for $\geq 4$ days

- PVAP: Isolation of significant count of a pneumonia pathogen from respiratory specimens such as tracheal aspirate, bronchoalveolar lavage, and sputum.

Data were analyzed using the GraphPad software (www.graphpad.com/quickcalcs/contingency2/) to calculate statistical significance between categorical data by Fisher's exact test and odds ratio for risk factor analysis. VAE rates were estimated per 1000 ventilator-days.

\section{RESULTS}

Over a period of 6 months, a total of 122 cases on MV were followed up. Out of the 122 cases, $16 \mathrm{VAE}$ cases were identified. The demographic profile and mortality of VAE cases were compared to those of non-VAE cases (patients who were on ventilator but did not develop any VAE) [Table 1]. No significant association was found between age, gender, mortality, and VAE cases. However, the mean total MV days and ICU days were very less for non-VAE cases (5.3 and 8.3, respectively) as compared to VAE cases (26.1 and 27.5, respectively). The overall VAE rate was 38.1/1000 MV days [Table 2]. Among VAE cases, $68.7 \%$ of patients expired, whereas $31.2 \%$ of patients survived. Among subtypes of VAE, $18.7 \%$ were VAC, $43.7 \%$ were IVAC, and $37.5 \%$ were PVAP. Survival rate was $100 \%$ for patients with VAC alone, whereas only $14.2 \%$ and $16.6 \%$ of patients survived with IVAC and PVAP, respectively.

The bacterial profile [Table 3] of respiratory samples was analyzed from both VAE and non-VAE cases. Among non-VAE cases, respiratory samples were cultured for only 31 cases,

\begin{tabular}{lccc}
\hline $\begin{array}{l}\text { Table 1: Demographic profile and mortality of ventilator- } \\
\text { associated event versus }\end{array}$ \\
cases
\end{tabular}

VAE: Ventilator-associated event, ICU: Intensive care unit, NS: Not significant, MV: Mechanical ventilation

Table 2: Ventilator-associated event incidence and mortality

\begin{tabular}{lcccc}
\hline & Total (\%) & $\begin{array}{c}\text { Rate (per 1000 } \\
\text { MV days) }\end{array}$ & \multicolumn{2}{c}{ Mortality, $\boldsymbol{n}$ (\%) } \\
\cline { 4 - 5 } & & & Survived & Expired \\
\hline VAE & $16(13.1)$ & 38.1 & $5(31.25)$ & $11(68.75)$ \\
VAC & $3(18.7)$ & 7.1 & $3(100)$ & 0 \\
IVAC & $7(43.7)$ & 16.7 & $1(14.2)$ & $6(85.7)$ \\
PVAP & $6(37.5)$ & 14.3 & $1(16.6)$ & $5(83.3)$ \\
\hline
\end{tabular}

VAC: Ventilator-associated condition, IVAC: Infection-related VAC, VAE: Ventilator-associated event, PVAP: Possible ventilator-associated pneumonia, MV: Mechanical ventilation

\begin{tabular}{|c|c|c|c|}
\hline $\begin{array}{l}\text { Total organism isolated } \\
(n=47)\end{array}$ & $\underset{(n=16)}{\mathrm{VAE}}$ & $\begin{array}{c}\text { Non-VAE } \\
(n=31)\end{array}$ & $P$ \\
\hline Polymicrobial & 4 & 7 & $1.0000(\mathrm{NS})$ \\
\hline Monomicrobial & 12 & 24 & \\
\hline Klebsiella spp. & 11 & 16 & $0.3547(\mathrm{NS})$ \\
\hline Pseudomonas spp. & 4 & 9 & $1.0000(\mathrm{NS})$ \\
\hline Acinetobacter spp. & 3 & 5 & $1.0000(\mathrm{NS})$ \\
\hline Escherichia coli & 0 & 2 & $0.5412(\mathrm{NS})$ \\
\hline Staphylococcus aureus & 1 & 1 & $1.0000(\mathrm{NS})$ \\
\hline Enterococcus spp. & 0 & 1 & $1.0000(\mathrm{NS})$ \\
\hline
\end{tabular}

NS: Not significant, VAE: Ventilator-associated event

whereas all 16 VAE cases had their respiratory samples cultured. No significant association was found between VAE and non-VAE cases with regard to bacterial isolate. The most common isolate was Klebsiella spp., followed by Pseudomonas 
spp. in both cases. Almost all strains were sensitive to quinolones, aminoglycosides, piperacillin-tazobactam, carbapenems, and colistin. Acinetobacter spp. were sensitive only to colistin and tigecycline. Respiratory isolates specifically from PVAP cases are shown in Table 4.

Depressed level of consciousness, immunosuppression, antibiotic use, and reintubation were significant risk factors associated with VAE cases [Table 5].

\section{Discussion}

In this study, the incidence and characteristics of VAE in ICU have been described and compared with that of the non-VAE cases. The patients in the ICU were nonsurgical and mainly had neurological disorders. The overall VAE rate in ICU reported in this study was $38.1 / 1000$ ventilator-days. This finding is very high as compared to a study done in a Japanese ICU, where the VAE rate was only $6.4 / 1000$ ventilator-days. ${ }^{[5]}$ Another study done by Zhu et al. reported a VAE rate of 11.1/1000 ventilator-days ${ }^{[6]}$ However, a study conducted in Kerala, India, also reported a high VAE rate of 29.2/1000 ventilator-days. ${ }^{[7]}$ Another study conducted in New Delhi reported a low VAE rate of $11.8 / 1000$ ventilator-days as compared to this study. ${ }^{[8]}$ Thomas et al. reported a VAE rate of 29.6/1000 ventilator-days in another study, also done in Kerala, India. ${ }^{[9]}$

Very few studies have been done in India using the new CDC VAE surveillance criteria. Most of the researches are on VAP rates (old surveillance criteria). The most common subtype of VAE reported in this study was IVAC (43.7\%). There was no significant association between mortality and VAE cases. Around $31 \%$ of mortality was seen in both categories. Other authors have reported that the presence of VAE was related to mortality and that higher mortality is seen in VAE cases compared to non-VAE cases. ${ }^{[6-8]}$ Among the subtypes of VAE,

\begin{tabular}{lc}
\hline $\begin{array}{l}\text { Table 4: Organisms isolated from respiratory specimens } \\
\text { of possible ventilator-associated pneumonia cases in our } \\
\text { intensive care units }\end{array}$ \\
\hline Organism & Total \\
\hline Klebsiella spp. & 3 \\
Acinetobacter spp. & 1 \\
Pseudomonas spp. & 2 \\
\hline
\end{tabular}

the survival rate for VAC alone subtype was $100 \%$, whereas only $14.2 \%$ and $16.6 \%$ of patients survived with IVAC and PVAP, respectively, in this study. The study conducted in a Japanese ICU, however, observed a tendency toward higher mortality with all the three subtypes, with no difference among them. ${ }^{[5]}$ Like mortality, no significant association was found between demographic profile and VAE cases. However, other studies have reported male gender to be significantly associated with VAE, whereas age had no significant association with VAE cases. ${ }^{[5,6]}$ In the present study, although mortality had no significant association with VAE, it was observed that the mean total MV days and ICU days were very less for non-VAE cases (5.3 and 8.3, respectively), whereas for VAE cases, it was 26.1 and 27.5 days, respectively. Similar findings have been reported by other studies also. ${ }^{[5-7]}$ This implies that VAE definitely increases the duration of hospital stay and as a consequence, increases the economic burden on the hospital and the patient.

The significant risk factors for VAE identified in this study were depressed level of consciousness, immunosuppression, antibiotic use, and reintubation $(P=0.0001)$. Lewis et al. in their research have found significant risk factors for VAE, especially VAC and IVAC. They observed that positive fluid balance, mandatory mode of ventilation, and use of drugs such as benzodiazepines, opioids, and muscle relaxants are risk factors for IVAC. ${ }^{[10]}$ Sedation and depressed level of consciousness increase the chances of reintubation and aspiration and prolong the duration of $\mathrm{MV}$, thus increasing the risk of VAE. ${ }^{[10,11]}$ Several other studies have also found that deep and sustained sedation, sedation with benzodiazepines and propofol, positive fluid balance, packed red blood cell transfusions, and high-tidal volume ventilation are all risk factors for VAE. ${ }^{[12-15]}$ Hence, in order to prevent VAEs, certain practices need to be followed in ICUs, which will help in minimizing the duration of $\mathrm{MV}$, avoiding intubation in patients and preventing conditions such as pneumonia, volume overload, and ARDS, leading to VAE. ${ }^{[3]}$

Klebsiella spp., Pseudomonas spp., and Acinetobacter spp. were the common bacterial isolates in the respiratory specimens of both VAE and non-VAE cases. The isolates were mostly sensitive to all antibiotics except Acinetobacter spp., which was sensitive only to colistin and tigecycline. Klebsiella spp. was the most common isolate in PVAP cases. Other studies

\begin{tabular}{|c|c|c|c|c|}
\hline Risk factor & VAE $(n=16)$ & Non-VAE $(n=106)$ & $P$ & OR $(95 \% \mathrm{Cl})$ \\
\hline Mean prolonged duration of ICU stay (days) & 18.5 & 5.6 & & \\
\hline Depressed level of consciousness & 14 & 25 & $0.0001(\mathrm{~S})$ & $22.68(4.8234-106.6422)$ \\
\hline Presence of chronic lung disease & 3 & 2 & $0.0160(\mathrm{~S})$ & $12(1.8314-78.6291)$ \\
\hline Prolonged use of antibiotic in the preceding 90 days & 12 & 10 & $0.0001(\mathrm{~S})$ & $28.8(7.8047-106.2740)$ \\
\hline Immunosuppression & 15 & 41 & $0.0001(\mathrm{~S})$ & $23.7805(3.0258-186.8939)$ \\
\hline Sedation & 11 & 55 & 0.4169 (NS) & $2.0400(0.6631-6.2756)$ \\
\hline Reintubation & 7 & 2 & $0.0001(\mathrm{~S})$ & $40.4444(7.2952-224.2231)$ \\
\hline
\end{tabular}

VAE: Ventilator-associated event, ICU: Intensive care unit, OR: Odds ratio, CI: Confidence interval 
have reported Acinetobacter spp. and Staphylococcus aureus as the most common organisms causing VAP. ${ }^{[16,17]}$ Most of them are multidrug-resistant pathogens. Magill et al. in their research have observed $S$. aureus, Pseudomonas aeruginosa, Klebsiella spp., and Escherichia coli as common pathogens causing PVAP. ${ }^{[18]}$ Out of these, $37.3 \%$ were methicillin-resistant S. aureus, 31.1\% were carbapenem-resistant Pseudomonas, and 14\% were carbapenem-resistant Klebsiella spp. Another study in India reported Acinetobacter spp. as a common isolate from VAE cases. ${ }^{[8]}$

\section{ConcLusion}

VAE increases cost of hospital stay with prolong MV and ICU days has been established. There is no significant difference with respect to age, gender, mortality, and respiratory isolate profile between VAE and non-VAE cases. Therefore, whether VAE should be considered a potential quality indicator for improving patient outcome in ICUs is uncertain, especially in ICUs where patients are at high risk due to comorbidities, immunosuppression, or depressed levels of consciousness and are expected to have a poor outcome. Larger, similar studies need to be done in order to assess the usefulness of such data for improving patient care and safety.

\section{Acknowledgment}

The authors would like to sincerely thank all the ICUs of the hospital for assisting in this study with special reference to nursing officers Sini and Dimple.

\section{Financial support and sponsorship}

Nil.

\section{Conflicts of interest}

There are no conflicts of interest

\section{REFERENCES}

1. Dudeck MA, Weiner LM, Allen-Bridson K, Malpiedi PJ, Peterson KD, Pollock DA, et al. National healthcare safety network (NHSN) report, data summary for 2012, device-associated module. Am J Infect Control 2013;41:1148-66.

2. Nora D, Póvoa P. Antibiotic consumption and ventilator-associated pneumonia rates, some parallelism but some discrepancies. Ann Transl Med 2017;5:450.

3. Klompas M. Potential strategies to prevent ventilator-associated events. Am J Respir Crit Care Med 2015;192:1420-30.
4. Magill SS, Klompas M, Balk R, Burns SM, Deutschman CS, Diekema $\mathrm{D}$, et al. Developing a new, national approach to surveillance for ventilator-associated events. Crit Care Med 2013;41:2467-75.

5. Nakahashi S, Imai H, Imanaka H, Ohshimo S, Satou T, Shima M, et al. Ventilator-associated events: Prevalence and mortality in Japan. J Thorac Dis 2018;10:6942-9.

6. Zhu S, Cai L, Ma C, Zeng H, Guo H, Mao X, et al. The clinical impact of ventilator-associated events: a prospective multi-center surveillance study. Infect Control Hosp Epidemiol 2015;36:1388-95.

7. Vaisakh G, Pavitharan S, Sigimol KM and Sruthimol VS. Incidence, risk factors and measures to prevent VAE among mechanically ventilated patients in selected ICUs of a tertiary care hospital, Kerala, India. Int J Adv Nur Management 2016;4:474-80.

8. Kumar S, Sen P, Gaind R, Verma PK, Gupta P, Suri PR, et al. Prospective surveillance of device-associated healthcare associated infection in an intensive care unit of a tertiary care hospital. Am J Infect Control 2018;46:202-6.

9. Thomas A, Jitendranath A, Vishwamohanan I, Bhai G, Sarika. Incidence of ventilator associated events among intubated patients in neurosurgery ICU of a tertiary health centre in India. Ind J Microbiology Research 2019;6:150-2.

10. Lewis SC, Li L, Murphy MV, Klompas M, CDC prevention epicenters. Risk factors for ventilator-associated events: A case-control multivariable analysis. Crit Care Med 2014;42:1839-48.

11. Jackson DL, Proudfoot CW, Cann KF and Walsh T. Systematic Review of the Impact of Sedation Practices in the ICU on Resource use, Cost and Patient Safety; 2010. Available From: http://www.biomedcentral. com/content/pdf/cc8956.pdf. [Last accessed on 2020 Jan 28].

12. Muscedere J, Sinuff T, Heyland DK, Dodek PM, Keenan SP, Wood G, et al. The clinical impact and preventability of ventilator associated conditions in critically ill mechanical ventilated patients. Chest 2013;144:1453-60.

13. Klompas M, Li L, Kleinman K, Szumita PM, Massaro AF. Associations between ventilator bundle components and outcomes. JAMA Intern Med 2016;176:1277-83.

14. Ogbu OC, Martin GS, Sevransky JE, Murphy DJ. High tidal volumes are independently associated with development of a ventilator associated condition in the ICU. Am J Respir Crit Care Med 2015;191:A3117.

15. Klompas M, Li L, Szumita P, Kleinman K, Murphy MV, CDC Prevention epicenters program. Associations between different sedatives and ventilator-associated events, length of stay, and mortality in patients who were mechanically ventilated. Chest 2016;149:1373-9.

16. Khurana S, Mathur P, Kumar S, Soni KD, Aggrawal R, Batra P, et al. Incidence of ventilator-associated pneumonia and impact of multidrug-resistant infections on patient's outcome: Experience at an apex trauma centre in North India. Indian J Med Microbiol 2017;35:504-10.

17. Chi SY, Kim TO, Park CW, Yu JY, Lee B, Lee HS, et al. Bacterial pathogens of ventilator associated pneumonia in a tertiary referral hospital. Tuberc Respir Dis (Seoul) 2012;73:32-7.

18. Magill SS, Li Q, Gross C, Dudeck M, Allen-Bridson K, Edwards JR. Incidence and characteristics of VAEs reported to the national healthcare safety network in 2014. Crit Care Med 2016;44:2154-62. 